\title{
The Parsec-Scale Morphology of Southern GPS Sources
}

\author{
P. G. Edwards ${ }^{1,3}$ and S. J. Tingay ${ }^{2}$ \\ ${ }^{1}$ Australia Telescope National Facility, CSIRO Astronomy and Space Science, Epping, NSW 1710, Australia \\ ${ }^{2}$ International Centre for Radio Astronomy Research, Curtin University, Bentley, WA 6102, Australia \\ ${ }^{3}$ Email: Philip.Edwards@csiro.au
}

(RECEIVED January 14, 2016; ACCEPTED November 11, 2016)

\begin{abstract}
Multi-frequency, multi-epoch ATCA observations of a sample of AGN resulted in the identification of nine new candidate Giga-hertz Peaked Spectrum sources. Here, we present Long Baseline Array observations at $4.8 \mathrm{GHz}$ of the four candidates with no previously published VLBI image, and consider these together with previously published VLBI images of the other five sources. We find core-jet or compact double morphologies dominate, with further observations required to distinguish between these two possibilities for some sources. One of the nine candidates, PKS 1831-711, displays appreciable variability, suggesting its GPS spectrum is more ephemeral in nature. We focus in particular on the apparent relationship between a narrow spectral width and 'compact double' parsec-scale morphology, finding further examples, but also exceptions to this trend. An examination of the VLBI morphologies high-redshift $(z>3)$ sub-class of GPS sources suggests that core-jet morphologies predominate in this class.
\end{abstract}

Keywords: galaxies: active - quasars: general

\section{INTRODUCTION}

GHz-Peaked Spectrum (GPS) sources are defined by a spectral turnover at $\mathrm{GHz}$ frequencies (O’Dea 1998). This class has been reported to represent at least $10 \%$ of the bright radio source population (O'Dea 1998) although the more recent study by Mingaliev et al. (2013) find that only $1 \sim 2 \%$ of a sample of $\sim 5000$ sources with $S_{5 \mathrm{GHz}}>200$ mJy met their criteria for classification as a GPS source. The catalogue of Labiano et al. (2007) contains 172 GPS sources, of which 156 have a flux density (generally, though not exclusively, at $5 \mathrm{GHz}$ ) above $200 \mathrm{mJy}$.

The class of GPS sources contains, amongst others, three distinct sub-classes: (i) radio galaxies, at low redshift, (ii) quasars at relatively high redshift, (iii) flaring active galactic nuclei which temporarily have a peaked spectrum during the spectral evolution of an outburst. Mingaliev et al. (2013) found a quarter of their sample consisted of blazars with spectra that may temporarily had a convex shape whilst the object was in an active state (see also Lister 2003; Kovalev 2005; Torniainen et al. 2008). Whilst all these meet the basic definition of a GPS source, we consider 'temporary' GPS sources to be a separate class from 'bona fide' or persistent GPS sources, and focus our interest on these.

In addition to the spectral criterion, all three sub-classes of GPS sources above are characterised by compact sub- kpc-scale radio structure. Bona fide GPS sources are further characterised by low radio polarisation, and low variability at radio wavelengths. An observed anti-correlation between turnover frequency and projected linear size (Fanti et al. 1990; O'Dea \& Baum 1997) has led to a model in which GPS sources evolve into Compact Steep Spectrum (CSS) sources, which are typically less than $20 \mathrm{kpc}$ in projected linear size, and possibly then into FR-I and/or FR-II sources (Snellen et al. 2003; Tingay \& Edwards 2015).

The identification of the GPS source PKS 2000-330 with a $z=3.77$ quasar by Peterson et al. (1982) - at the time most distant source known-led O'Dea (1990) to consider the prevalence of GPS quasars at high redshift. Although the population of known $z>3$ sources at the time was not large, O'Dea found that about half of the known $z>3$ quasars were GPS sources and additionally that about half of the then known GPS quasars lay in this redshift range. More recently, Coppejans et al. (2015) identified a sample of 33 megahertz peaked-spectrum (MPS) sources and determined redshifts for 24 , with an average redshift of 1.3. Five of the sources had $z>2$, with four fainter sources for which redshifts could not be found, thought to be at even higher redshifts, suggesting that the MPS sources are also good candidates for highredshift sources. In contrast, however, Mingaliev et al. (2013) reported that there was a deficit of objects at large redshifts with peak frequencies below several $\mathrm{GHz}$. 
Early studies of the parsec-scale morphology of GPS source found that many could be characterised as a 'compact double', with two components of comparable flux density separated by up to some tens of milliarcseconds. Stanghellini (2003) concluded that GPS quasars tend to have core-jet (CJ) or complex morphologies whereas GPS galaxies were more likely to be compact symmetric objects (CSO).

Data from a multi-frequency, multi-epoch Australia Telescope Compact Array (ATCA) survey (Tingay et al. 2003a) was used to search for new GPS sources (Tingay, Edwards, \& Tzioumis 2003b; Edwards \& Tingay 2004). The survey, which was made in conjunction with the VSOP Survey Program (Hirabayashi et al. 2000), originally contained 212 sources; however, 17 of these were dropped after the first few epochs as it was clear they did not meet the criteria for inclusion in the VSOP Survey. The remaining 185 sources were observed at up to 16 epochs between 1996 and 2000 at $1.4,2.5,4.8$, and $8.6 \mathrm{GHz}$. The multi-epoch observations allowed determination of the variability index, $M$, defined as the r.m.s. variation from the mean flux density, divided by the mean flux density. Examination of these data resulted in the identification of nine new Southern Hemisphere GPS candidates (Tingay et al. 2003b; Edwards \& Tingay 2004). In this paper, we combine new LBA observations at $4.8 \mathrm{GHz}$ of the four southernmost GPS candidates with published images of the other five sources to study the parsec-scale morphologies and the sizes of the compact components in these candidate GPS sources, and consider the implications for the sub-categories of GPS sources listed above. In particular, we examine the correlation between narrow spectral width and compact double morphology suggested by Edwards \& Tingay (2004), and the morphology of the high redshift $(z>3)$ sub-class of GPS sources for insights into the nature of these sources.

\section{SOURCE CHARACTERISTICS}

We list in Table 1 the source classification and redshift, and the $4.8 \mathrm{GHz}$ mean flux density, $S_{5}$, mean fractional polarisation, $p_{5}$, and variability index, $M_{5}$, from Tingay et al. (2003a). The width is the FWHM of the fitted spectrum in decades of frequency (see Edwards \& Tingay 2004 for details).

The mean variability index at $5 \mathrm{GHz}$ for the 185 sources ranged between 0.01 and 0.43 , with a median value of 0.08 (see Tingay et al. 2003a for tabulated values and distributions). The highest observed linear polarisation was $8.19 \%$ at $5 \mathrm{GHz}$, with a median level of $2.2 \%$. Twenty-four sources (13\% of the total) had polarisation levels below the reliably measurable level of $0.5 \%$. The nine new GPS candidates identified by Edwards \& Tingay (2004) showed the expected low variability and low fractional polarisation characteristic of the class.

Here, we describe LBA observations of the four southernmost sources: PKS 1619-680 (J1624-6809), MRC 1722644 (J1726-6427), PKS 1831-711 (J1837-7108), and PKS 2146-783 (J2152-7807). The other five candidates from Ed- wards \& Tingay (2004) have declinations north of $-40^{\circ}$ and the results of Very Long Baseline Array (VLBA) observations of these sources have been reported previously. Four of the five were observed at $5 \mathrm{GHz}$ as part of the VSOP Pre-Launch Survey (VLBApls: Fomalont et al. 2000), a companion programme to the ATCA programme to derive the final source list for the VSOP AGN Survey (Hirabayashi et al. 2000), and the fifth source was studied by Tingay \& Edwards (2015).

\section{LBA OBSERVATIONS}

The southernmost four GPS candidates were observed with the Australian Long Baseline Array (Edwards \& Phillips 2015) over an 18-h period starting on 2003 November 26 at 20:00 UT (proposal code v170). The observing schedule cycled around the four sources such that each source was observed for approximately one quarter of the time but over the full time range of the observation. In addition to the target sources, PKS 1921-293 (approximately $9.4 \mathrm{Jy}$ at this time for our observing frequency) and PKS 1610-771 (approximately $3.7 \mathrm{Jy}$ at this time for our observing frequency) were observed for short periods as fringe-finders and to assist in establishing the flux density calibration for the observations. These flux densities were obtained by interpolating between observations in that ATCA Calibrator Database ${ }^{1}$.

The array of telescopes consisted of the ATCA $(5 \times 22 \mathrm{~m})$, Mopra (22 m), Parkes (64 m), Hobart (26 m), and Ceduna $(30 \mathrm{~m})$. The $\mathrm{S} 2$ recording system was used to record a $16-\mathrm{MHz}$ band in left and right circular polarisations, centred at $4.816 \mathrm{GHz}$. The recorded data were correlated at the Australia Telescope National Facility S2 correlator (Wilson, Roberts, \& Davis 1996). Due to instrumental issues at Ceduna, the data from this telescope was not usable and is not considered further.

The correlated data were imported into AIPS ${ }^{2}$. As all the target sources are bright, phase reference calibrators were not included in the observing schedule. Fringe-fitting proceeded on the fringe-finders and targets, finding good delay and rate solutions for each antenna across the full time range and for both polarisations. The fringe-fit solutions were applied to the data. System temperature data and gain information from each antenna were imported into AIPS and applied to the correlated data to convert the amplitudes from correlation coefficients into jansky. We examined the calibrated data for PKS 1921-293 and estimated corrections to the antenna gains by comparison to the catalogued flux density for PKS 1921293 at $4.8 \mathrm{GHz}$ near the observing epoch. These corrections were then applied to all target sources, via a second round of amplitude calibration in AIPS.

The data for all target sources were then exported as UVFITS files and further examined using Difmap (Shepherd 1997). In Difmap, the data for each target was flagged for

\footnotetext{
${ }^{1}$ http://www.narrabri.atnf.csiro.au/calibrators/

${ }^{2}$ AIPS is produced and maintained by the National Radio Astronomy Observatory, a facility of the National Science Foundation operated under cooperative agreement by Associated Universities, Inc.
} 
Table 1. GPS candidates from Edwards \& Tingay (2004). Flux density at $4.8 \mathrm{GHz}, S_{5}$, variability index at $4.8 \mathrm{GHz}, M_{5}$, fractional polarisation at $4.8 \mathrm{GHz}, p_{5}$, and peak frequency $v_{\mathrm{pk}}$, are reproduced from Edwards \& Tingay (2004). LAS is the largest angular size inferred from the VLBI images considered in this paper. See text for details.

\begin{tabular}{|c|c|c|c|c|c|c|c|c|c|c|}
\hline $\begin{array}{l}\text { Source } \\
\text { name }\end{array}$ & $\mathrm{J} 2000$ & $z$ & $\begin{array}{c}v_{\mathrm{pk}} \\
(\mathrm{GHz})\end{array}$ & width & $\begin{array}{l}M_{5} \\
(\%)\end{array}$ & $p_{5}$ & $\begin{array}{c}S_{5} \\
(\mathrm{Jy})\end{array}$ & $\begin{array}{c}S_{\text {VLBI }} \\
(\mathrm{Jy})\end{array}$ & $\begin{array}{l}\text { LAS } \\
\text { (mas) }\end{array}$ & $\begin{array}{l}\text { LAS } \\
\text { pc }\end{array}$ \\
\hline PKS 0150-334 & J0153-3310 & 0.610 & 1.5 & 1.6 & 0.04 & 1.35 & 0.88 & 0.86 & 1.0 & 6.8 \\
\hline PKS 0434-188 & J0437-1844 & 2.702 & 4.5 & 1.3 & 0.05 & 0.80 & 0.95 & 1.08 & 1.1 & 9.1 \\
\hline PKS 0642-349 & J0644-3459 & 2.165 & 3.3 & 1.4 & 0.09 & 2.03 & 0.85 & 0.84 & 3.4 & 28.7 \\
\hline PKS 1619-680 & J1624-6809 & 1.360 & 3.1 & 1.4 & 0.04 & $<0.5$ & 1.69 & 1.56 & 4.0 & 34.2 \\
\hline MRC 1656-075 & J1658-0739 & 3.742 & 4.8 & 1.2 & 0.03 & $<0.5$ & 1.32 & 1.36 & 7.1 & 51.7 \\
\hline MRC 1722-644 & J1726-6427 & $\ldots$ & 1.1 & 1.0 & 0.02 & $<0.5$ & 1.26 & 1.36 & 47.0 & $\ldots$ \\
\hline PKS 1831-711 & J1837-7108 & 1.356 & 8.2 & 2.0 & 0.06 & 1.77 & 2.39 & 3.40 & 13.4 & 114.5 \\
\hline PKS 2146-783 & $\mathrm{J} 2152-7807$ & 3.997 & 4.3 & 1.3 & 0.03 & $<0.5$ & 1.15 & 1.39 & 0.4 & 2.6 \\
\hline PKS 2254-367 & $\mathrm{J} 2257-3627$ & 0.006 & 2.7 & 1.8 & 0.04 & $<0.5$ & 1.28 & 1.15 & 83.0 & 10.3 \\
\hline
\end{tabular}

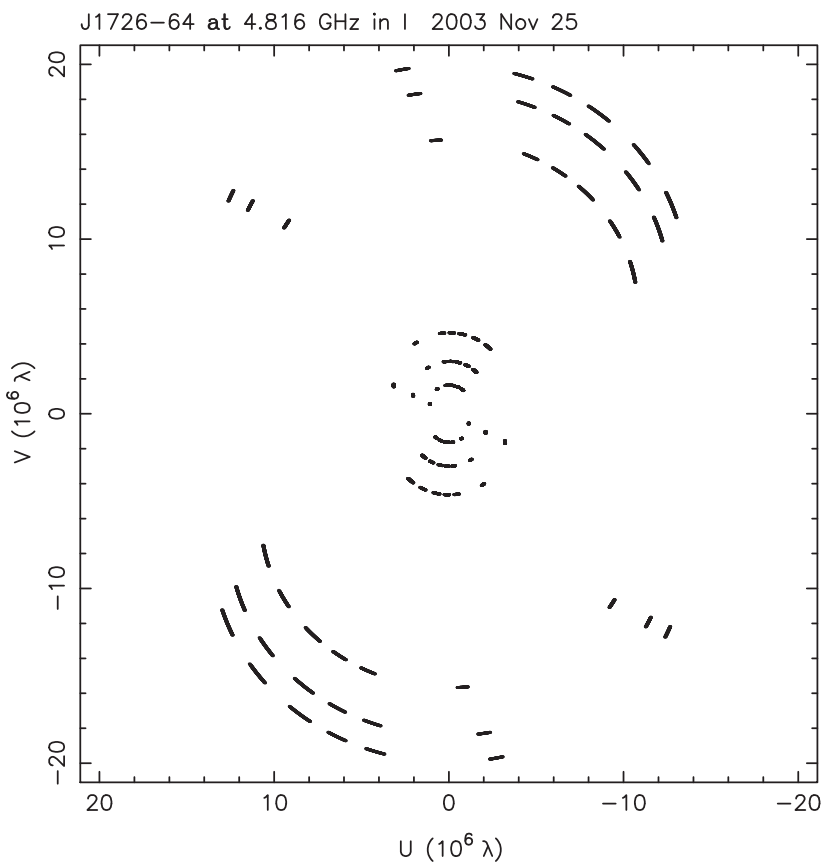

Figure 1. The $(u, v)$ coverage for MRC 1722-644 (J1726-6427) with Parkes, ATCA, Mopra, and Hobart The scans were typically 15 min in duration.

bad visibilities, averaged over 10-s periods (the correlation integration time was $2 \mathrm{~s}$ ), and averaged the two circular polarisations into Stokes I. As an example, the $(u, v)$ coverage for MRC 1722-644 (J1726-6427) is shown in Figure 1. Eaach target was imaged (images sizes of $128 \times 128$ pixels of 3 mas pixel size) and calibrated using standard clean and selfcalibration tasks. Deconvolution was guided by clean windows, set on the peak residuals in the field, and cleaning proceeded until the peak negative and peak positive residuals had comparable magnitudes. Both phase and amplitude self-calibration was employed, with overall amplitude selfcalibration corrections to the flux densities of order $10 \%$ for all antennas and all targets. We adopt $10 \%$ as the error on the

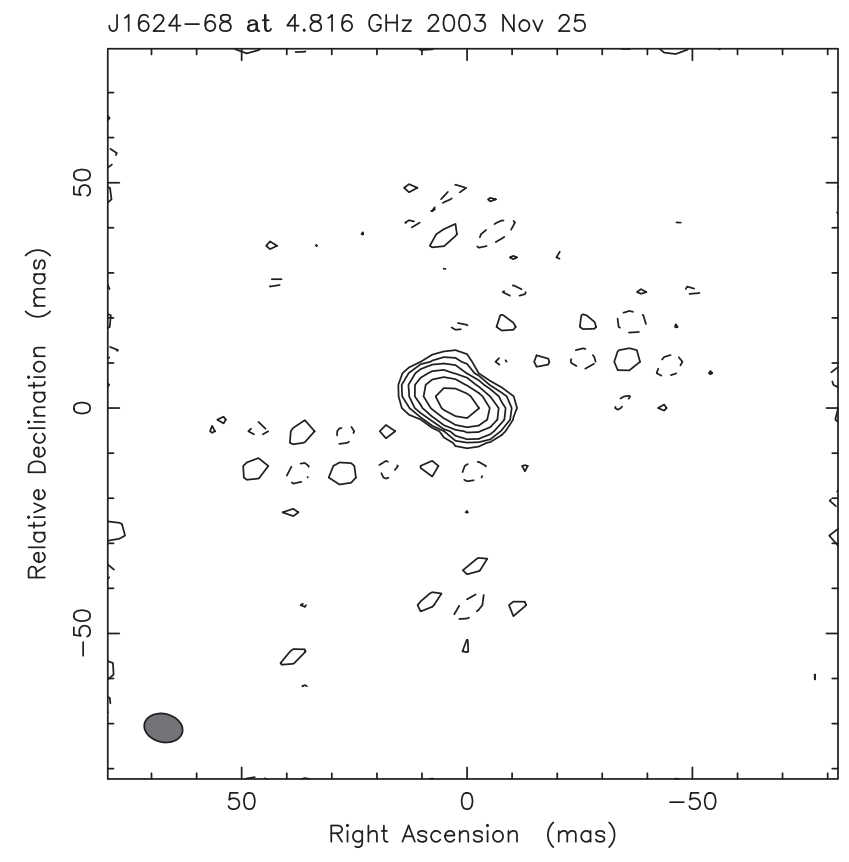

Figure 2. LBA image of PKS 1619-680 (J1624-6809). The image peak is $0.99 \mathrm{Jy} / \mathrm{beam}$, with contours at $-2,2,4,8,16,32$, and $64 \% \mathrm{t}$ of the peak. The beam FWHM is 8.6 mas $\times 6.3$ mas at a position angle of $77^{\circ}$.

flux density estimates guided by previous experience (e.g., Tingay et al. 1998). The angular resolution achieved in the images ranged between 6 and 23 mas (Figures 2-5). The typical image r.m.s. achieved was approximately $10 \mathrm{mJy}$, producing images with dynamic ranges of approximately 50 to 100.

In order to extract quantitative information on the target source morphologies, at the end of the imaging and selfcalibration process, we replaced the point source clean component models with elliptical Gaussian components, between one and three for each target source. The Difmap task modelfit was then used to fit the models to the data for each target. The results of the model fitting are given in Table 2. 
Table 2. Parameters of the target source structure models (elliptical Gaussian model components). $S$ is the total flux density at $4.8 \mathrm{GHz}$ of the model component in Jy. $r$ is the angular distance of the model component from the image phase centre in milliarcseconds. $\theta$ is the position angle of the model component relative to the phase centre, in degrees east of north. $a$ is the full width at half maximum of the major axis of the model component in milliarcseconds. $b$ is the ratio of the minor axis to the major axis full widths at half maximum. $\phi$ is the position angle of the major axis of the model component, east of north.

\begin{tabular}{lcccccrr}
\hline \hline Source & J2000 & $\begin{array}{c}S \\
(\mathrm{Jy})\end{array}$ & $\begin{array}{c}r \\
(\mathrm{mas})\end{array}$ & $\begin{array}{c}\theta \\
(\mathrm{deg})\end{array}$ & $\begin{array}{c}a \\
(\mathrm{mas})\end{array}$ & $\begin{array}{c}\phi \\
(\mathrm{deg})\end{array}$ \\
\hline PKS 1619-680 & $\mathrm{J} 1624-6809$ & 0.71 & 5.1 & 56 & 6.7 & 0.35 & 55 \\
& & 0.85 & 0.9 & 80 & 5.4 & 0.00 & 66 \\
MRC 1722-644 & $\mathrm{J} 1726-6427$ & 0.53 & 6.4 & -2 & 6.1 & 0.54 & 46 \\
& & 0.36 & 2.5 & 90 & 5.7 & 0.25 & 82 \\
PKS 1831-711 & $\mathrm{J} 1837-7108$ & 0.47 & 40.8 & 141 & 5.1 & 0.26 & 49 \\
& & 2.3 & 0.1 & -62 & 2.1 & 0.64 & -18 \\
PKS 2146-783 & $\mathrm{J} 2152-7807$ & 1.1 & 13.3 & 142 & 7.7 & 0.00 & 65 \\
& 1.39 & 0.0 & -26 & 0.36 & 1.0 & -169 \\
\hline \hline
\end{tabular}

\section{RESULTS}

In this section, we present the results of our LBA observations and consider published observations of the other sources and attempt to characterise the parsec-scale morphology of the source. We are guided by the work of Snellen, Schilizzi, \& van Langevelde (2000) who classified the radio morphologies as CJ sources, CSO, Compact Double sources (CD), or Complex sources. CD may be considered a subset of CSO (see, e.g., Peck \& Taylor 2000): A CD source has no central component, whereas a CSO has a central component with extended structure on both sides.

PKS 0150-334: Imaged at $5 \mathrm{GHz}$ as part of the VLBApls observations in 1996 June and resolved into two components of similar brightness separated by 1 mas (Fomalont et al. 2000). Whilst at face value this is suggestive of a compact double, the spacing is somewhat less than would be typical for this class (cf. Snellen et al. 2000) and a CJ morphology cannot be ruled out. This is borne out by the subsquent observation at $8.4 \mathrm{GHz}$ by Ojha et al. (2004) in 2002 November, which revealed two components separated by 3.3 mas. Acknowledging that a speed derived from only two observations should be treated with caution, at a redshift of 0.61, an apparent motion of 2.3 mas in 6.4 yrs corresponds to an apparent superluminal motion of $12.8 c$. Further observations are required to confirm this but, in any case, it is apparent that the source has a CJ morphology.

PKS 0434-188: Imaged as part of the VLBApls (Fomalont et al. 2000) and resolved into two components of similar brightness (0.62 and $0.45 \mathrm{Jy}$ ) separated by 1.1 mas. The source was observed in 2002 February as part of the VSOP Survey Program with the resulting model containing two components ( 0.62 and $0.35 \mathrm{Jy}$ ) separated by 1.3 mas (Dodson et al. 2008). (We note an error in the formatting of Table 3 of Dodson et al. which has resulted in a third component being erroneously listed for this source-closer inspection indicates it is actually the second component of J0743-6726.) In the light of the previous example, further observations are required to better characterise the source evolution, both spectral and morphological, and distinguish between a compact double or CJ morphology.

PKS 0642-349: The VLBApls image (Fomalont et al. 2000 ) is dominated by a compact core of $0.69 \mathrm{Jy}$ and a secondary component of $0.15 \mathrm{Jy}$ located 3.4 mas to the west, with a possible third component a further 10 mas to west. This has the characteristics of a CJ morphology.

PKS 1619-680: Our LBA image (Figure 2) reveals two components of similar flux density and with similar major axes separated by 7 mas. We note the modelfit to the data presented in Table 2 has a linear (formally zero-width) second component: We do not interpret this literally but rather take it to suggest that an elogated Gaussian component would provide an acceptable fit, and that a higher resolution observation may resolve this component into a number of smaller components. Higher resolution observations are required to distinguish between a compact double morphology or a $\mathbf{C J}$ morphology.

MRC 1656-075: The VLBApls image is dominated by a bright, resolved $1.3 \mathrm{Jy}$ component, with a $0.05 \mathrm{Jy}$ secondary offset by 7.1 mas (Fomalont et al. 2000). The disparity in flux densities argues against classification as a compact doublePeck \& Taylor (2000), for example, adopted a requirement of a ratio less than 10:1 for a CSO_-and suggests a CJ morphology. Multi-frequency data to examine the spectral characteristics of the components would be useful.

MRC 1722-644: Our LBA observations resolve this source into two components separated by almost 40 mas (Figure 3). The brighter of these is more extended, and can be modelled by two overlapping components. This morphology is reminiscent of PKS 1934-638 (Tzioumis et al. 2002; Tzioumis et al. 2010), and we categorise it as a compact double. Dodson et al. (2008) note that in a VSOP Survey observation there were no detections on space baselines.

PKS 1831-711: Our LBA observations resolve this source into two components separated by 13 mas (Figure 4). The brighter component is about twice as bright as the fainter, 
Table 3. GPS sources with redshifts, $z>3$, from O'Dea (1990), Labiano et al. (2007) and this work. Morphologies are U (unresolved) or CJ (core-jet). LAS is the approximate largest angular size (see text for details).

\begin{tabular}{|c|c|c|c|c|c|}
\hline B1950 & $\mathrm{J} 2000$ & $z$ & Morphology & $\begin{array}{l}\text { LAS } \\
\text { (mas) }\end{array}$ & Reference \\
\hline $0201+113$ & J0203+1134 & 3.639 & CJ & 2 & Pushkarev \& Kovalev (2012) \\
\hline 0420-388 & J0422-3844 & 3.110 & $\ldots$ & $\ldots$ & $\ldots$ \\
\hline $0636+680$ & J0642+6758 & 3.180 & $\mathrm{U}$ & $\ldots$ & Britzen et al. (2007), Pushkarev \& Kovalev (2012) \\
\hline $0642+449$ & $\mathrm{~J} 0646+4451$ & 3.396 & $\mathrm{CJ}$ & 9 & Gurvits et al. (1992) \\
\hline $1351-018$ & J1354-0206 & 3.707 & $\mathrm{CJ}$ & 13 & Frey et al. (1997) \\
\hline $1354-174$ & J1357-1744 & 3.147 & $\mathrm{CJ}$ & 32 & Frey et al. (1997) \\
\hline $1422+231$ & $\mathrm{~J} 1424+2256$ & 3.626 & $\mathrm{CJ}$ & $\ldots$ & Orienti et al. (2006) \\
\hline $1442+101$ & $\mathrm{~J} 1445+0958$ & 3.535 & $\mathrm{CJ}$ & 22 & Pushkarev \& Kovalev (2012) \\
\hline $1526+670$ & $\mathrm{~J} 1526+6650$ & 3.020 & $\mathrm{CJ}$ & 2 & Britzen et al. (2007) \\
\hline $1614+051$ & $\mathrm{~J} 1616+0459$ & 3.197 & $\mathrm{CJ} ?$ & 2 & Orienti et al. (2006), Pushkarev \& Kovalev (2012) \\
\hline $1656-075$ & J1658-0739 & 3.742 & $\mathrm{CJ}$ & 7 & this work \\
\hline $1839+389$ & $\mathrm{~J} 1840+3900$ & 3.095 & $\mathrm{CJ}$ & 2 & Britzen et al. (2007) \\
\hline $2000-330$ & J2003-3251 & 3.773 & $\mathrm{CJ}$ & 2 & Ojha et al. (2005) \\
\hline $2126-158$ & J2129-1538 & 3.270 & CJ & 2 & Pushkarev \& Kovalev (2012) \\
\hline $2146-783$ & J2152-7807 & 3.997 & $\mathrm{U}$ & $\ldots$ & this work \\
\hline
\end{tabular}

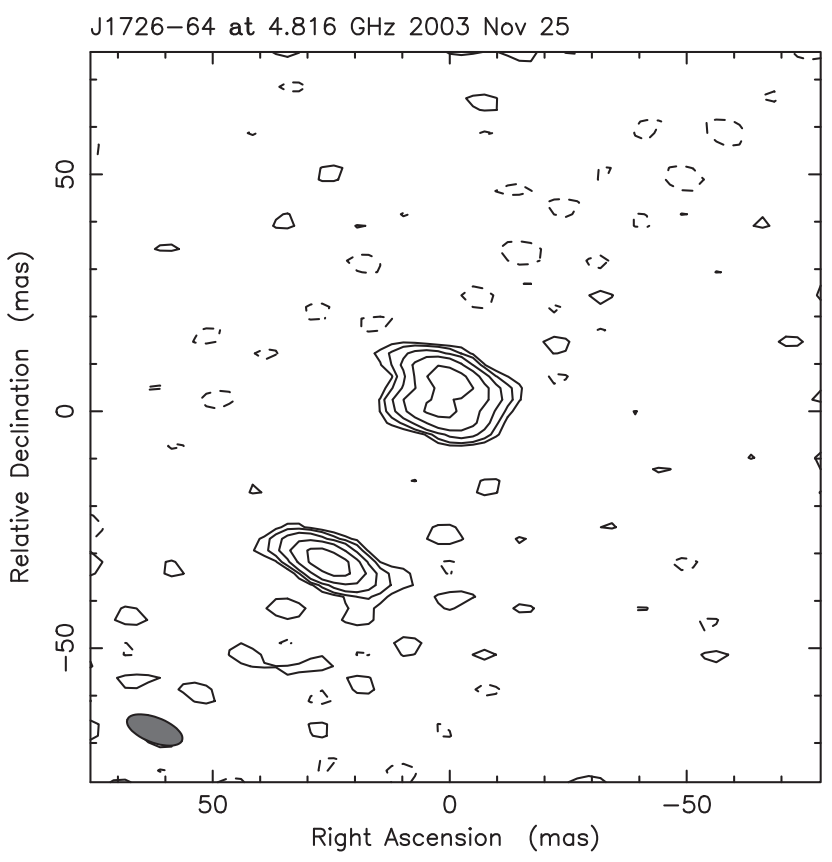

Figure 3. LBA image of MRC 1722-644 (J1726-6427). The image peak is $0.42 \mathrm{Jy} / \mathrm{beam}$, with contours at $-4,4,8,16,32$, and $64 \%$ of the peak. The beam FWHM is 12.4 mas $\times 5.3$ mas at a position angle of $69^{\circ}$.

with the latter being more extended. The best modelfit to the more extended component is a single linear component which we consider in the same manner as for PKS 1619-680. Based on the image alone, we would provisionally classify this as a compact double; however, as discussed in the next section, consideration of additional information leads us to favour a $\mathrm{CJ}$ interpretation. We note that a VSOP survey observation in 1999 May found a compact core, 0.2 mas in size, and with evidence of some extended structure (Dodson et al. 2008), but

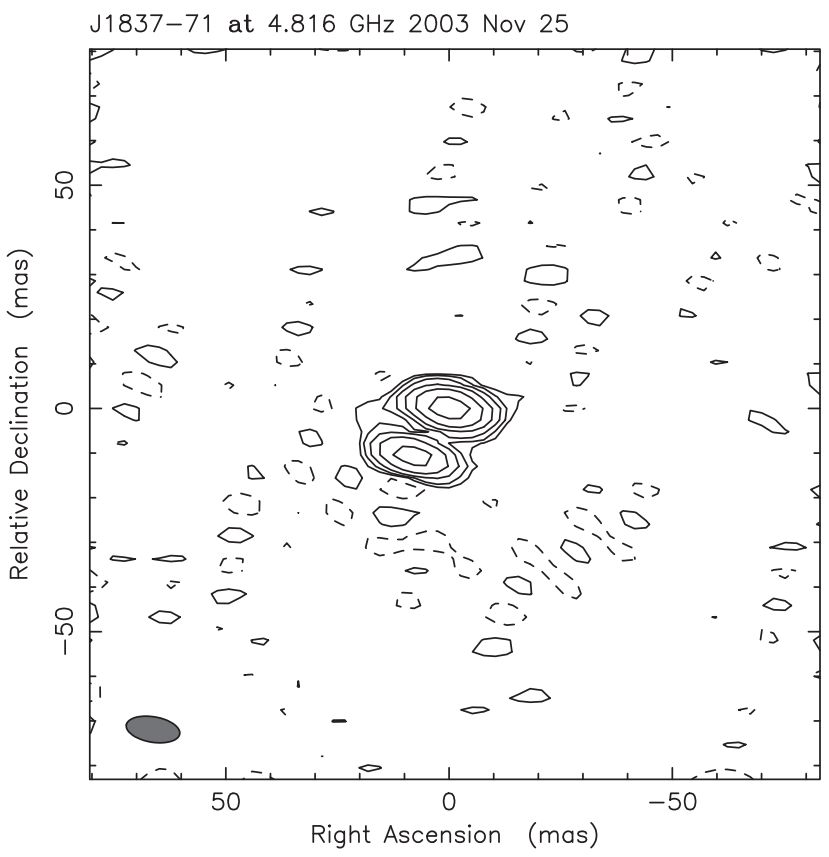

Figure 4. LBA image of PKS 1831-711 (J1837-7108). The image peak is $2.22 \mathrm{Jy} / \mathrm{beam}$, with contours at $-2,2,4,8,16,32$, and $64 \%$ of the peak. The beam FWHM is 12.1 mas $\times 5.7$ mas at a position angle of $80^{\circ}$.

suggest the limited ground array in that observation (Hobart, Mopra, Hartebeesthoek) may have struggled to resolve the structure revealed by our observation.

PKS 2146-783: Our LBA observations yield a strongly core-dominated source, modelled by a single circular Gaussian component (Figure 5). Despite this, Dodson et al. (2008) found no detections on space baselines in a VSOP survey observation. Based on our observations, the source is unresolved. 


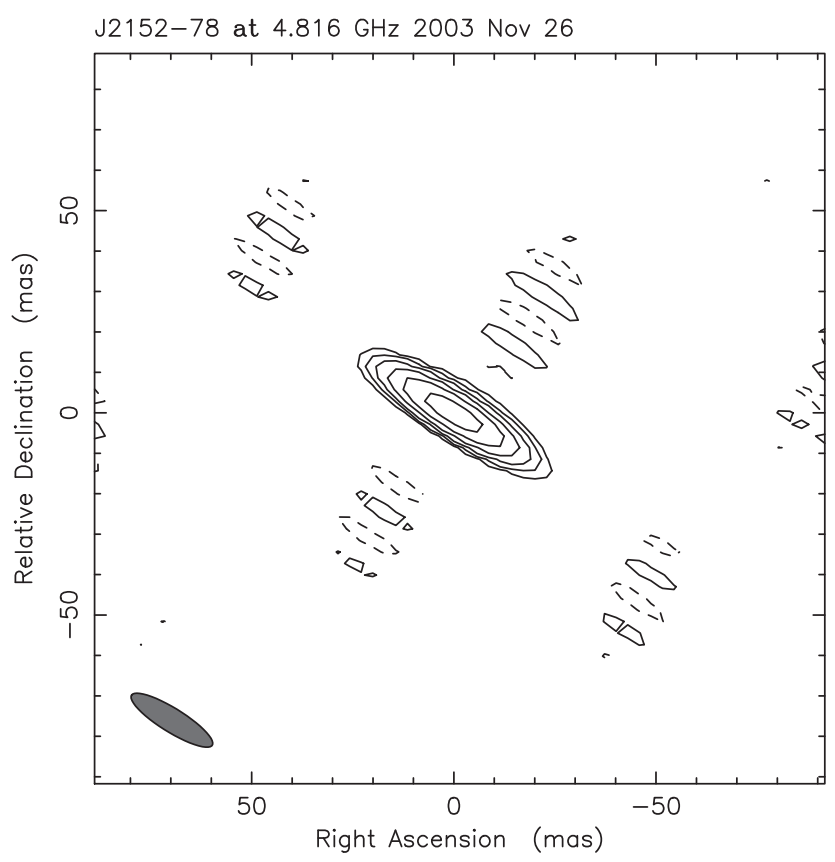

Figure 5. LBA image of PKS 2146-783 (J2152-7807). The image peak is $1.39 \mathrm{Jy} / \mathrm{beam}$, with contours at $-2,2,4,8,16,32$, and $64 \%$ of the peak. The beam FWHM is 23.5 mas $\times 5.9$ mas at a position angle of $58^{\circ}$.

PKS 2254-367: This low-redshift GPS source was imaged at four frequencies with the VLBA in 2003 November, revealing a compact core and symmetric structure on both sides, leading to classification as a CSO (Tingay \& Edwards 2015).

\section{DISCUSSION}

\subsection{Status of Southern GPS candidates}

In Table 1, we give the sum of the flux densities of the components in the VLBI image and compare this to the mean flux density in the ATCA monitoring. We note these measurements are non-contemporaneous (VLBApls in June 1996; ATCA monitoring 1996 to 2000; our LBA observations 2003) but as bona fide GPS sources display generally low variability this is not as critical as might otherwise be the case. It is apparent that the VLBI observations have recovered $\gtrsim 90 \%$ of the flux density in all cases. The 'missing' flux density may be due to low-level extended emission, or to source variability.

The biggest discrepancy is for PKS 1831-711, where the VLBI flux density signficantly exceeds the ATCA flux density. Edwards \& Tingay (2004) previously noted that the PKSCAT flux densities for this source, e.g., $1.15 \mathrm{Jy}$ at $5.0 \mathrm{GHz}$ (Wright \& Otrupcek 1990), differ significantly from more recent values, e.g., $2.29 \mathrm{Jy}$ (Wright et al. 1994) and $2.39 \mathrm{Jy}$ (Tingay et al. 2003a), suggesting significant long-term variability. The total flux density of the components in the LBA image, $3.4 \mathrm{Jy}$, is higher again, confirming this source is sig- nificantly more variable than the majority of GPS sources. (Its ATCA variability index was the second highest of the nine candidates here.) Edwards \& Tingay (2004) also pointed out that the spectral width of J1837-7108 was the largest of all nine candidates. This is further evidence in favour of a $\mathrm{CJ}$ interpretation of the morphology, and suggests that the source belongs to the class of AGN which only temporarily have a peaked spectrum during the spectral evolution of an outburst.

Similarly, Edwards \& Tingay (2004) inferred moderate long-term variability for J2152-7807 from differences between PKSCAT values, e.g., $0.77 \mathrm{Jy}$ at $5 \mathrm{GHz}$ (Wright \& Otrupcek 1990), and more recent determinations, $1.13 \mathrm{Jy}$ (Wright et al. 1994) and $1.15 \mathrm{Jy}$ (Tingay et al. 2003a).

Table 1 also contains the largest angular size (LAS) of the sources based on the VLBI observations. The conversion from angular to projected linear size was made using the cosmology calculator of Wright (2006) adopting $H_{0}=$ $69.6 \mathrm{~km} \mathrm{~s}^{-1} \mathrm{Mpc}^{-1}, \Omega_{\mathrm{M}}=0.286$, and $\Omega_{\mathrm{vac}}=0.714$. The values of the LAS confirm that the milli-arcsecond scale structure (which, as noted above, makes up the overwhelming majority of the radio emission) is confined within a kiloparsec in all cases, as expected for GPS sources.

\subsection{Distribution of morphological types}

We have classified the parsec-scale morphologies as four likely CJ sources, one CSO, one compact double, one unresolved, and two which may be compact doubles or CJ sources. Further observations of these latter two, ideally at two or more frequencies to determine the spectral characteristics of the components, would enable their classifications to be determined.

Although our sample is small, it is interesting to compare it with the results of Snellen et al. (2000). For their sample of 47 faint GPS sources (with peak flux densities ranging between 30 and $900 \mathrm{mJy}$ ), three were classified as CSO, 11 as $\mathrm{CD}$, seven as $\mathrm{CJ}$, and two as complex. Of the remainder, 20 could not be classified as they were too compact. Snellen et al. (2000) noted, as we have, that many of the sources classified as $\mathrm{CD}$ or $\mathrm{CJ}$ had only two components, resulting in their classifications being tentative. The biggest difference between our results and those of Snellen et al. is that we have far fewer unresolved sources, most likely as the sources in our sample are brighter, with peak flux densities $\gtrsim 1 \mathrm{Jy}$.

Stanghellini et al. (1997), Stanghellini, O’Dea, \& Murphy (1999) considered the morphological information available for a sample of 20 GPS sources and found that GPS quasars have preferentially CJ or complex morphologies and GPS galaxies tend to be CSOs. Only one of our candidates is classified as a galaxy, and it is a CSO. We find less evidence for complex morphologies amongst the quasars; however, our sample is small.

We note that PKS 0642-349, which has a clear CJ morphology, has the highest fractional polarisation (2\%) and highest variability index (0.09) of the nine candidates. 
PKS 0150-334, which also has a CJ morphology, similarly has a detectable fractional polarisation.

\subsection{High-redshift sources}

Edwards \& Tingay (2004) considered the redshift distribution of the GPS sources and candidates and confirmed it differed significantly from that of a larger flat-spectrum sample. The trend has become even stronger for this sample of nine candidates, as two of the three sources without redshift determinations at the time have subsequently been identified by Healey et al. (2008) with $z>3$ quasars. (The other, MRC 1722-644, still has no redshift.)

Mingaliev et al. (2013) report that there is a deficit of objects at large redshifts with peak frequencies below several GHz. Inspection of Table 1 appears to support this, as the $z>2$ sources all have peak frequencies greater than $3 \mathrm{GHz}$; however, comparison with the larger sample of sources in Table 1 of Edwards \& Tingay (2004) reveals this apparent trend is not as evident when a larger sample (albeit smaller than that of Mingaliev et al.) is considered.

In Table 3, we compile a list of GPS sources and canididates with $z>3$ from O'Dea (1990), Labiano et al. (2007) and this work, in order to examine the parsec-scale morphologies of this class of extreme GPS sources. The age of universe spanned by these redshifts corresponds to 1.6 to 2.2 Gyr. B0429-388 is the only source for which there is not a published VLBI image (and we note that whilst the spectrum is curved it has yet to be firmly established that it is in fact peaked). We tabulate the approximate LAS of the parsec-scale image, noting that the VLBI images span a range of sensitivities due to both the epoch the observations were made (more recent observations are generally made with wider bandwidths) and the observing mode (some are snapshots, others are dedicated observations). We do not give a LAS for $\mathrm{B} 1422+231$ as it is gravitational lensed and the lensed images are subject to a range of amplifications in flux density and scale, though none of the images is larger than several mas in extent (Orienti et al. 2006).

Only two of the sources are unresolved, and several extend over more than 10 mas. The resolved sources all appear to have a CJ morphology: Orienti et al. (2006) proposed B1614+051 as a candidate CSO source; however, comparison with the subsequent observation of Pushkarev and Kovalev (2012) lead us to tentatively favour a CJ morphology. This predominance of CJ morphologies is in accord with previous reports that quasars are more likely to have $\mathrm{CJ}$ or complex structures (Stanghellini 2003).

We note that the sources in Table 3 are compiled from several sources and are subject to their respective selection biases, and reiterate that the VLBI morphologies are based on a heterogeneous set of VLBI observations. Nevertheless, the predominance of $\mathrm{CJ}$ morphologies, and absence of $\mathrm{CD}$ or CSO morphologies, is striking and deserves a more systematic follow-up.

\subsection{The spectral widths of Compact Doubles}

Edwards \& Tingay (2004) observed that the spectrum of MRC 1722-644 has a width comparable to the (narrow) spectra of the compact double PKS 1934-638 (Tzioumis et al. 2002; Tzioumis et al. 2010) and the CSO 0108+388 (O'Dea, Baum, \& Stanghellini 1991; Marr, Taylor, \& Crawford 2001) which led them to predict that MRC 1722-644 might also display a Compact Double morphology, which is borne out by our LBA observations.

Further examples can be found in the sample of Dallacasa et al. (1998): 1518+047 and 1607+268 are Compact Doubles with separations of 150 and 60 mas, respectively, and both have spectral widths of $\sim 1.1$ decades of frequency. More recently, Callingham et al. (2015) reported that the GPS source PKS B0008-421 has the smallest known spectral width of any GPS source, and the steepest known spectral slope below the turnover, close to the theoretical limit of synchrotron self-absorption. The VLBI-scale morphology has two components separated by $\sim 120$ mas.

Simplistically, such a correlation seems reasonable: If CD sources are comprised of two lobes, with the central component either heavily absorbed (at these frequencies) or in a quiescent state, it would be quite natural for the superposition of the spectra of the two components to be narrower than the case where an additional, generally flatter spectrum, core component also contributes to the total source spectrum. However, inspection of other CD sources indicates that any such correlation is not tight: Taylor \& Peck (2003) found PKS 2344-192 (J2347-1856) to be a compact double with a separation of $\sim 32$ mas, but the source has yet to be established as a GPS source (whilst the spectrum is curved, it is not clear it is peaked). Similarly, Taylor et al. (2000) found 1031+567 to be a compact double with a separation of $\sim 34$ mas. Whilst archival data indicates it has a peaked spectrum, the width is $\sim 1.5$ decades of frequency, appreciably broader than the examples above.

Generally, however, sources with CD or CSO morphologies must either be viewed close to side-on, or possess jets where doppler boosting does not play a significant role, if we are indeed seeing two lobes of similar brightness. Strong absorption of the core suggests we are viewing the source side-on, with the absoption in a disc or torus of material surrounding the core. In contrast, in CJ sources-assuming jets are intriscially two-sided - we are seeing the doppler boosted jet oriented towards us and not seeing the doppler deboosted jet travelling in the opposite direction (although again, absorption could play a role close to the core). Variability is a common feature of CJ sources, amplified by Doppler boosting, and so one might expect $\mathrm{CJ}$ sources generally to be more variable, and therefore for the spectra of $\mathrm{CJ}$ sources to evolve away from having GPS spectra more quickly than their CSO or $\mathrm{CD}$ counterparts. $\mathrm{CD}$ sources might then be expected to be less (or more slowly) variable, as is the case for $1031+567$ (Fassnacht \& Taylor 2001) and PKS 1934-638, the primary flux density calibrator for the ATCA (Partridge et al. 2016). 
MRC 1722-644, which we classify as a CD, has the lowest variability index of the nine candidates (see Table 1).

\section{FUTURE WORK}

We have established that one of the candidate GPS sources examined shows evidence for sufficient variability to exclude it as a bona fide GPS source. The other sources remain good candidates for inclusion in GPS catalogues. Multi-epoch, and multi-frequency, VLBI observations in the future would enable the morphologies of the sources to be established more firmly, particular in discriminating between $\mathrm{CJ}$ sources and CD sources.

Ongoing montoring to establish the persistence (or otherwise) of the peaked spectra is another approach, the utility of which has been demonstrated for PKS 1718-649 by Tingay et al. (2015). In addition, results at lower frequencies from the MWA GLEAM survey (Wayth et al. 2015; Hurley-Walker et al. 2017) and the Australian SKA Pathfinder (Johnston et al. 2007) will extend the frequency range over which the spectra can be determined and monitored.

\section{CONCLUSIONS}

We have presented the first parsec-scale images of four candidate southern GPS sources and considered these alongside published VLBI images of the five other candidate GPS sources from Edwards \& Tingay (2004). We find CJ or compact double morphologies dominate, with further observations required to distinguish between these two possibilities. The sole CSO, PKS 2254-367, is a nearby galaxy. One of the nine candidates, PKS 1831-711, displays appreciable variability, suggesting its GPS spectrum is more ephemeral in nature. Although the sample is small, there is some evidence that $\mathrm{CJ}$ sources are more polarised and more variable, and that compact doubles are the least variable. MRC 1722-644, which has the narrowest spectral width of the sample, has a compact double morphology, and whilst there are other examples of such a correlation it is not universal. Two of our sample have been found to lie amongst the highest redshift GPS sources known. An examination of the VLBI morphologies of the 15 catalogued high-redshift $(z>3)$ sub-class of GPS sources suggests that CJ morphologies predominate in this class, with more systematic follow-up of this apparent trend encouraged.

\section{ACKNOWLEDGEMENTS}

The Long Baseline Array is part of the Australia Telescope National Facility which is funded by the Commonwealth of Australia for operation as a National Facility managed by CSIRO. This research has made use of NASA's Astrophysics Data System, and the NASA/IPAC Extragalactic Database (NED) which is operated by the Jet Propulsion Laboratory, California Institute of Technology, under contract with the National Aeronautics and Space Administration. We thank Dave Jauncey, John Reynolds, Tasso Tzioumis, and Jim Lovell in particular for their efforts in operating and maintaining the LBA at the time these observations were made.

\section{REFERENCES}

Britzen, S., et al. 2007, A\&A, 472, 763

Callingham, J. R., et al. 2015, ApJ, 809, 168

Coppejans, R., Cseh, D., Williams, W. L., van Velzen, S., \& Falcke, H. 2015, MNRAS, 450, 1477

Dallacasa, D., Bondi, M., Alef, W., \& Mantovani, F. 1998, A\&AS, 129,219

Dodson, R., et al. 2008, ApJS, 175, 314

Edwards, P. G., \& Phillips, C. J. 2015, PKAS, 30, 659

Edwards, P. G., \& Tingay, S. J. 2004, A\&A, 424, 91

Fanti, R., et al. 1990, A\&A, 231, 333

Fassnacht, C. D., \& Taylor, G. B. 2001, AJ, 122, 1661

Fomalont, E. B., et al. 2000, ApJS, 131, 95

Frey, S., Gurvits, L. I., Kellermann, K. I., Schilizzi, R. T., \& Pauliny-Toth, I. I. K. 1997, A\&A, 325, 511

Gurvits, L. I., et al. 1992, A\&A, 260, 82

Healey, S. E., et al. 2008, ApJS, 175, 97

Hirabayashi, H., et al. 2000, PASJ, 52, 997

Hurley-Walker, N., Callingham, J. R., Hancock, P. J., et al. 2017, MNRAS, 464, 1146

Johnston, S., et al. 2007, PASA, 24, 174

Kovalev, Y. Y. 2005, BaltA, 14, 413

Labiano, A., et al. 2007, A\&A, 463, 97

Lister, M. L. 2003, in ASP Conf. Proc., Vol. 300, Radio Astronomy at the Fringe, eds. J. A. Zensus, M. H. Cohen, \& E. Ros (San Francisco: ASP), 71

Marr, J. M., Taylor, G. B., \& Crawford, F., III 2001, ApJ, 550, 160

Mingaliev, M. G., Sotnikova, Y. V., Mufakharov, T. V., Erkenov, A. K., \& Udovitskiy, R. Y. 2013, AstBu, 68, 262 and erratum 68,494

O’Dea, C. P. 1990, MNRAS, 245, 20P

O'Dea, C. P. 1998, PASP, 110, 493

O’Dea, C. P., \& Baum, S. A. 1997, AJ, 113, 148

O’Dea, C. P., Baum, S. A., \& Stanghellini, C. 1991, ApJ, 380, 66

Ojha, R., et al. 2004, AJ, 127, 3609

Ojha, R., et al. 2005, AJ, 130, 2529

Orienti, M., Dallacasa, D., Tinti, S., \& Stanghellini, C. 2006, A\&A, 450,959

Partridge, B., et al. 2016, ApJ, 821, 61

Peck, A. B., \& Taylor, G. B. 2000, ApJ, 534, 90

Peterson, B. A., Savage, A., Jauncey, D. L., \& Wright, A. E. 1982, ApJ, 260, L27

Pushkarev, A. B., \& Kovalev, Y. Y. 2012, A\&A, 544, A34

Shepherd, M. C. 1997, ADASS VI, 125, 77

Snellen, I. A. G., Schilizzi, R. T., \& van Langevelde, H. J. 2000, MNRAS, 319, 429

Snellen, I. A. G., et al. 2003, PASA, 20, 38

Stanghellini, C. 2003, PASA, 20, 118

Stanghellini, C., et al. 1997, A\&A, 325, 943

Stanghellini, C., O’Dea, C. P., \& Murphy, D. W. 1999, A\&AS, 134, 309

Taylor, G. B., Marr, J. M., Pearson, T. J., \& Readhead, A. C. S. 2000, ApJ, 541, 112

Taylor, G. B., \& Peck, A. B. 2003, ApJ, 597, 157

Tingay, S. J., et al. 1998, AJ, 115, 960

Tingay, S. J., et al. 2003a, PASJ, 55, 351 
Tingay, S. J., \& Edwards, P. G. 2015, MNRAS, 448, 252

Tingay, S. J., Edwards, P. G.\& Tzioumis, A. K. 2003b, MNRAS, 346,327

Tingay, S. J., et al. 2015, AJ, 149, 74

Torniainen, I., et al. 2008, A\&A, 482, 483

Tzioumis, A., et al. 2002, A\&A, 392, 841

Tzioumis, A. K., et al. 2010, AJ, 140, 1506

Wayth, R. B., et al. 2015, PASA, 32, e025
Wilson, W. E., Roberts, P. P., \& Davis, E. R. 1996, in Proc. 4th AsiaPacific Telecope Workshop, ed. E. A. King (Sydney: CSIRO ATNF), 16

Wright, A., \& Otrupcek, R. 1990, PKS Catalog (Sydney: CSIRO Australia Telescope National Facility)

Wright, A. E., Griffith, M. R., Burke, B. F., \& Ekers, R. D. 1994, ApJS, 91, 111

Wright, E. L. 2006, PASP, 118, 1711 